\title{
LA INFLUENCIA DE LAS EMOCIONES EN LA ELECCIÓN DE CARRERAS UNIVERSITARIAS
}

\author{
Ana Belén Borrachero Cortés \\ Departamento de Psicología de la Educación y Psicobiología. Facultad de Educación. Universidad Internacional \\ de La Rioja, España. Email: belenborrachero@hotmail.com \\ María Antonia Dávila Acedo \\ Departamento de Didáctica de las Ciencias Experimentales y de las Matemáticas. Facultad de Educación. \\ Universidad de Extremadura. Badajoz, España. \\ Diego Airado Rodríguez \\ Departamento de Didáctica de las Ciencias Experimentales y de las Matemáticas. Facultad de Educación. \\ Universidad de Extremadura. Badajoz, España. \\ https://doi.org/10.17060/ijodaep.2017.n1.v2.925
}

Fecha de Recepción: 4 Febrero 2017

Fecha de Admisión: 1 Abril 2017

\section{RESUMEN:}

Con el fin de conocer las emociones que experimentaron los estudiantes de Educación Secundaria en el aprendizaje de diferentes asignaturas científicas y cómo ha podido influir en la posterior elección de carreras universitarias, realizamos una investigación con 142 estudiantes del Máster Universitario en Formación del Profesorado de Educación Secundaria (MUFPES) de la rama de Ciencias durante tres cursos académicos consecutivos (2010/2013), los cuales se encuentran distribuidos en tres especialidades científicas ofertadas en el Máster: Física/Química, Biología/Geología y Matemáticas. El estudio realizado nos lleva a afirmar que existe una concordancia entre las emociones experimentadas como aprendices de ciencias y las futuras carreras realizadas con posterioridad, destacando las emociones positivas por encima de las emociones negativas.

DESCRIPTORES: emociones, elección de carreras, formación del profesorado, ciencias, Educación Secundaria.

\section{ABSTRACT:}

The influence of emotions in the choice of university careers

In order to know the emotions experienced by secondary school students in learning different scientific subjects and how they could influence the subsequent choice of university careers, we carried out an investigation with 142 students from Master's Degree in Teacher Training in Secondary Education (MUFPES) of the branch of Sciences during three courses (2010/2013), which are distributed in three scientific specialties offered in the Master: Physics / Chemistry, Biology / Geology and Mathematics. The study leads us to affirm that there is a concordance between the emotions 


\section{LA INFLUENCIA DE LAS EMOCIONES EN LA ELECCIÓN DE CARRERAS UNIVERSITARIAS}

experienced as science learners and future careers carried out later, emphasizing positive emotions above negative emotions.

KEY WORDS: emotions, careers choice, teacher training, science, Secondary Education.

\section{ANTECEDENTES.}

Hasta hace poco, los componentes afectivos estaban infravalorados en la educación, existiendo una desconexión latente entre las dimensiones cognitivas y afectivas en el proceso de enseñanza y aprendizaje (Sutton y Wheatley, 2003). En la actualidad, cada vez existen más trabajos que interrelacionan lo cognitivo con lo afectivo (Hargreaves, 2000; Shapiro, 2010), argumentando la idea de que la afectividad y la cognición se comprenden mejor cuando se consideran como funciones mentales independientes y complementarias (Frijda, 2000).

Ciertamente, el ámbito afectivo es uno de los aspectos más estudiados dentro del campo de la psicología y la educación, que va teniendo mayor impacto en la investigación en didáctica de las ciencias (Abrahams, 2009; Marbá y Márquez, 2010) y más concretamente en la enseñanza de las ciencias (Barmby y Defty, 2006; Mellado, Blanco, Borrachero y Cárdenas, 2013), centrándose en las creencias, actitudes y emociones.

Uno de los problemas más pronunciados en la educación científica es la connotación de dificultad y aburrimiento que conllevan los contenidos de ciencias, emociones negativas, provocando una huida de estudiantes a otros itinerarios no científicos, sobre todo cuando llega el momento de la elección de carrera universitaria (Fensahm, 2004; Rocard et al., 2007).

Con el paso del tiempo, la ciencia escolar va creando una imagen negativa en la mente de los escolares; de hecho, Ios propios alumnos describen la ciencia como autoritaria, aburrida, difícil 0 irrelevante para la vida diaria (Vázquez y Manassero, 2008). Asimismo, Solbes (2011) detalla cómo los estudiantes de secundaria ven las asignaturas de ciencia como aburridas, excesivamente teóricas, poco útiles y difíciles. Al mismo tiempo, se señala un descenso de estudiantes que eligen cursar una asignatura dentro de la temática científica. Otras causas que podrían influir son el profesor, la falta de trabajo práctico o la excesiva orientación para preparar los exámenes en las clases (Murphy y Beggs, 2003).

Diversas investigaciones concluyen que las emociones hacia las ciencias son diferentes según la materia a aprender (Borrachero y Brígido, 2011; Brígido, Bermejo, Conde, Borrachero y Mellado, 2010; Costillo et al., 2010). Las asignaturas de Ciencias Naturales (Biología y Geología) y Tecnología registran emociones positivas como diversión, satisfacción, tranquilidad, placer,... En cambio, las materias de Física, Química y Matemáticas presentan un aumento de emociones negativas como nerviosismo, preocupación, ansiedad, tensión, etc., disminuyendo a su vez las emociones positivas. Además, se desprende la idea de que las emociones hacia las ciencias van cambiando a la vez que aumenta la edad de los alumnos, pues las emociones negativas son más notables en la etapa de Educación Secundaria que en los ciclos de Educación Primaria.

No cabe duda de que las variables afectivas influyen intensamente en la elección de las futuras vocaciones (Fouad, 2007). Es importante tener en cuenta que es en Educación Secundaria donde los estudiantes comienzan a elegir sus futuros itinerarios educativos dependiendo significativamente de la formación que reciben y los contenidos curriculares, en tanto en cuanto estos pueden servir para desarrollar un sinfín de componentes afectivos: la curiosidad, el interés y el gusto por las ciencias, 0 de forma contraria, desarrollar el aburrimiento, la dificultad y el fracaso, provocando el desinterés y el rechazo hacia las ciencias (Vázquez y Manassero, 2009). 


\section{OBJETIVOS.}

Con este trabajo nos fijamos dos objetivos principales:

Conocer las emociones que experimentaron los futuros profesores de Educación Secundaria en el aprendizaje de las materias científicas en la misma etapa.

Encontrar una posible influencia de las emociones experimentadas en Educación Secundaria con la elección de la carrera universitaria.

\section{PARTICIPANTES.}

El muestreo probabilístico incidental o de conveniencia es el proceso metodológico utilizado para seleccionar la muestra de estudio, pues son los sujetos de la Universidad de Extremadura quienes nos brindan un acceso más fácil. También, conocemos al profesorado que imparte docencia en el MUFPES, lo que nos permite utilizar la hora lectiva para realizar el estudio. Además, evitamos el tener que desplazarnos fuera de la comunidad autónoma de Extremadura.

La muestra, con la que vamos a trabajar, está constituida por un total de 142 estudiantes del MUFPES de la rama de Ciencias durante tres cursos académicos consecutivos (2010/2013), los cuales se encuentran distribuidos en tres especialidades científicas ofertadas en el Máster: Física/Química, Biología/Geología y Matemáticas. La distribución muestral por especialidad y curso académico aparece en la Tabla 1.

Tabla 1.

Distribución de la muestra por especialidad y curso académico.

\begin{tabular}{|c|c|c|c|}
\hline Especialidad & $2010 / 2011$ & 2011/2012 & $2012 / 2013$ \\
\hline $\begin{array}{c}\text { Física/Química } \\
\mathrm{N}=39 \\
27,5 \%\end{array}$ & $\begin{array}{c}18 \text { sujetos } \\
30,5 \%\end{array}$ & $\begin{array}{c}14 \text { sujetos } \\
26,9 \%\end{array}$ & $\begin{array}{c}7 \text { sujetos } \\
26,6 \%\end{array}$ \\
\hline $\begin{array}{c}\text { Biología/Geología } \\
\mathrm{N}=68 \\
47,9 \%\end{array}$ & $\begin{array}{c}25 \text { sujetos } \\
42,4 \%\end{array}$ & $\begin{array}{c}27 \text { sujetos } \\
51,9 \%\end{array}$ & $\begin{array}{c}16 \text { sujetos } \\
51,6 \%\end{array}$ \\
\hline $\begin{array}{c}\text { Matemáticas } \\
\mathrm{N}=35 \\
24,6 \%\end{array}$ & $\begin{array}{c}16 \text { sujetos } \\
27,1 \%\end{array}$ & $\begin{array}{c}11 \text { sujetos } \\
21,2 \%\end{array}$ & $\begin{array}{c}8 \text { sujetos } \\
25,8 \%\end{array}$ \\
\hline $\begin{array}{c}\text { Total } \\
\mathrm{N}=142 \\
100,0 \%\end{array}$ & $\begin{array}{c}59 \text { sujetos } \\
100,0 \%\end{array}$ & $\begin{array}{c}52 \text { sujetos } \\
100,0 \%\end{array}$ & $\begin{array}{c}31 \text { sujetos } \\
100,0 \%\end{array}$ \\
\hline
\end{tabular}

En la Tabla 2 vemos las frecuencias y porcentajes de la muestra distribuidas según el género del sujeto. De esta forma, sabemos que más de la mitad de la muestra total $(62,7 \%)$ es de género femenino. Distinguiendo por especialidad, también sucede que, en Biología/Geología y Matemáticas (64,7\% y 74,3\% respectivamente), la mayoría son mujeres, mientras que en Física/Química la diferencia se encuentra en que los hombres representan el $51,3 \%$ de la muestra en el total de dicha especialidad. 


\section{LA INFLUENCIA DE LAS EMOCIONES EN LA ELECCIÓN DE CARRERAS UNIVERSITARIAS}

Tabla 2.

Distribución de los futuros profesores de Secundaria por género.

\begin{tabular}{l|cc|cc|rr|rr}
\hline & \multicolumn{2}{|c|}{$\mathrm{B} / \mathrm{G}$} & \multicolumn{2}{c|}{$\mathrm{F} / \mathrm{Q}$} & \multicolumn{2}{c|}{ Mat } & \multicolumn{2}{c}{ Global } \\
\hline & $\mathrm{n}$ & $\%$ & $\mathrm{n}$ & \multicolumn{1}{c|}{$\%$} & \multicolumn{1}{c}{$\mathrm{n}$} & \multicolumn{1}{c}{$\%$} & $\mathrm{n}$ & $\%$ \\
\hline \hline Hombre & 24 & 35,3 & 20 & 51,3 & 9 & 25,7 & 53 & 37,3 \\
Mujer & 44 & 64,7 & 19 & 48,7 & 26 & 74,3 & 89 & 62,7 \\
Total & 68 & 100,0 & 39 & 100,0 & 35 & 100,0 & 142 & 100,0 \\
\hline \hline
\end{tabular}

\section{MÉTODO}

Al considerar que la metodología cuantitativa es la más idónea para nuestro estudio, procedimos a elaborar un cuestionario que cubriera las necesidades de nuestra investigación. El cuestionario es una herramienta de recogida de información considerado clásico en las ciencias sociales para la obtención y recogida de datos. Se define como un instrumento basado en una serie de preguntas acerca de un determinado problema sobre el que se desea investigar y cuyas respuestas han de contestarse por escrito (Del Rincón, Latorre y Sanz, 1995).

Para este estudio, realizamos una clasificación de emociones basadas en las emociones que más se repetían tras la revisión de las clasificaciones de varios investigadores importantes en la temática: la clasificación de emociones primaras y secundarias de Goleman (1996); la clasificación de emociones básicas de Casacuberta (2000); la clasificación de emociones primarias y secundarias de Fernández-Abascal, Martín y Domínguez (2001); la escala de bienestar y malestar emocional de Rebollo, García, Barragán, Buzón y Vega (2008); la clasificación de emociones básicas de Bisquerra (2009): y, por último, la clasificación de emociones universales, de fondo y sociales de Damasio (2010).

Dicha revisión concluyó con la elección de ocho emociones positivas y ocho emociones negativas (Tabla 3). Cada emoción en cada una de las cinco materias científicas fue codificada como variable dicotómica con los valores sí o no, dando lugar a 80 variables.

Tabla 3.

Clasificación de emociones.

\begin{tabular}{|c|c|c|c|}
\hline : & $\begin{array}{l}\text { Alegría } \\
\text { Confianza } \\
\text { Diversión } \\
\text { Orgullo } \\
\text { Placer } \\
\text { Satisfacción } \\
\text { Simpatía } \\
\text { Tranquilidad }\end{array}$ & 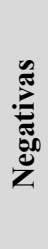 & $\begin{array}{l}\text { Aburrimiento } \\
\text { Ansiedad } \\
\text { Desesperación } \\
\text { Irritabilidad } \\
\text { Nerviosismo } \\
\text { Odio } \\
\text { Pesimismo } \\
\text { Preocupación }\end{array}$ \\
\hline
\end{tabular}

El análisis descriptivo se utilizó para recopilar y presentar los datos del cuestionario, utilizando la distribución por puntuación media y su expresión gráfica, mientras que el análisis inferencial se efectuó en función de la naturaleza de las variables a tratar y de los grupos de contraste, siempre comprobando que se cumplían los supuestos de Normalidad, Aleatorización y Homocedasticidad de la varianza, para realizar pruebas paramétricas o no. 
La Prueba Chi-Cuadrado, también conocida como Ji-Cuadrado, nos permite contrastar si las frecuencias observadas en cada una de las opciones de una variable categórica varían de forma significativa de las frecuencias que se esperarían encontrar si la muestra hubiese sido extraída de una población con una determinada distribución de frecuencias. Por tanto, es la alternativa idónea para contrastar y encontrar la asociación entre dos variables categóricas.

\section{RESULTADOS.}

El estudio realizado pretende encontrar diferencias en las emociones según la especialidad del MUFPES que cursan los sujetos, presumiendo que si la materia científica coincide con la especialidad encontraremos un incremento de emociones positivas. Por tanto, se divide en 3 sub-apartados, una por cada especialidad.

Las diferencias entre las especialidades se representaran en gráficos de barras. Las emociones positivas se encuentran a la izquierda y las emociones negativas a la derecha, medidas en porcentaje de sujetos de la especialidad del Máster que señalan haber experimentado la emoción. Cada color representa una especialidad.

\section{Emociones en el aprendizaje de la Biología y la Geología.}

Como cabría esperar, un mayor porcentaje de sujetos de la especialidad de Biología/Geología manifiesta haber experimentado emociones positivas en el aprendizaje de contenidos de Biología (ESO). Asimismo, encontramos un menor porcentaje de sujetos que experimenta emociones negativas, a diferencia de las otras especialidades de Física/Química y Matemáticas (Figura 1).

Figura 1.

Porcentaje de futuros profesores que experimentaron emociones en el aprendizaje de Biología según la especialidad del Máster cursada.

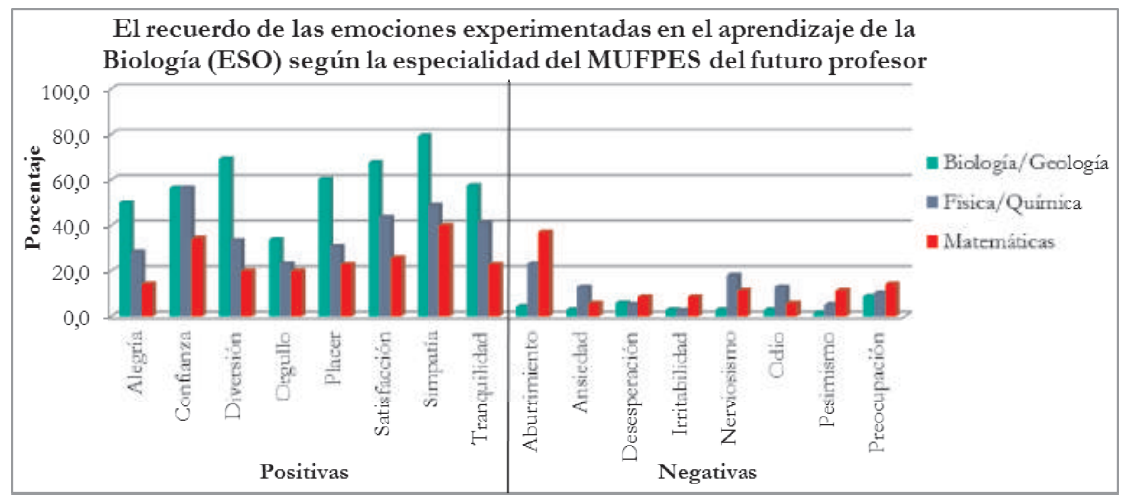

Como vemos en la Figura 2, a excepción de las emociones confianza y placer, son los sujetos de la especialidad de Biología/Geología quienes experimentaron en mayor porcentaje emociones positivas en el aprendizaje de Geología (ESO), a diferencia de las otras dos especialidades. También vemos como en las emociones negativas obtienen menores porcentajes. 


\section{LA INFLUENCIA DE LAS EMOCIONES EN LA ELECCIÓN DE CARRERAS UNIVERSITARIAS}

Figura 2.

Porcentaje de futuros profesores que experimentaron emociones en el aprendizaje de Geología según la especialidad del Máster cursada.

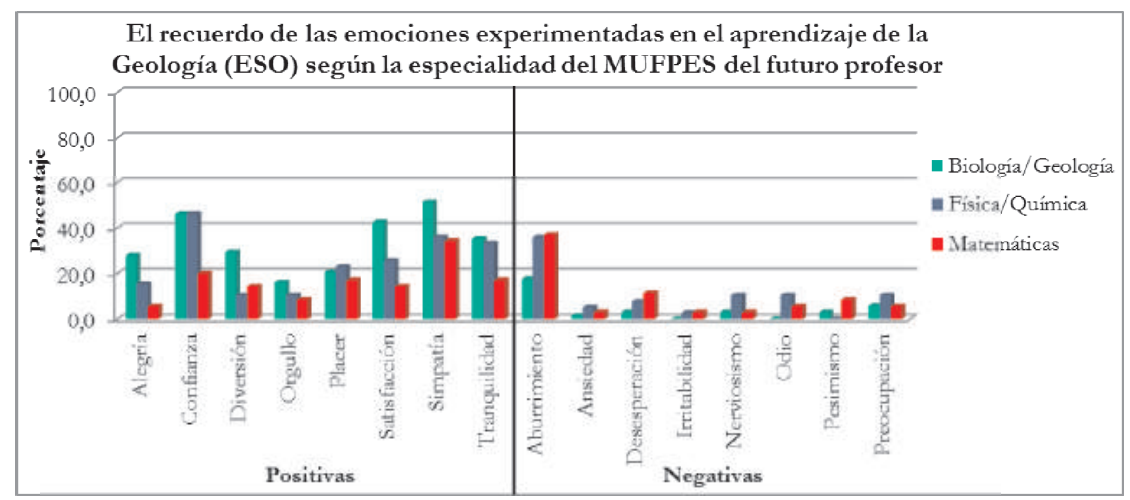

En la Tabla 4 se recoge los resultados obtenidos tras realizador la Prueba Chi-Cuadrado entre las emociones experimentadas en el aprendizaje de Biología y Geología y las tres especialidades del MUFPES que cursa la muestra.

Encontramos diferencias significativas $(p \leq, 050)$ en casi todas las emociones positivas y solamente en las emociones negativas aburrimiento $(p>, 000)$ y nerviosismo $(p=, 030)$ experimentadas en el aprendizaje de la Biología (ESO). Con estos datos interpretamos que son los futuros profesores de la especialidad de Biología/Geología quienes presentan mayor probabilidad de haber experimentado emociones positivas en el aprendizaje de la Biología de Educación Secundaria y menor probabilidad de haber experimentado aburrimiento y nerviosismo.

En el aprendizaje de la Geología encontramos diferencias significativas, entre las tres especialidades del Máster, en las emociones alegría $(p=, 020)$, diversión $(p=, 036)$, satisfacción $(p=, 009)$, aburrimiento $(p=, 042)$ y odio $(p=, 035)$. Así, podemos decir que los sujetos de la especialidad de Biología/Geología experimentaron en mayor medida emociones positivas (alegría, diversión y satisfacción) y en menor medida emociones negativas (aburrimiento y odio). 
Tabla 4.

Prueba Chi-Cuadrado de la relación especialidad de Máster y el recuerdo de emociones experimentadas en el aprendizaje de Biología y Geología.

\begin{tabular}{|c|c|c|c|c|c|}
\hline & & \multicolumn{2}{|l|}{ Biología } & \multicolumn{2}{|c|}{ Geología } \\
\hline & & Chi-Cuadrado & Sig. & Chi-Cuadrado & Sig. \\
\hline \multirow{8}{*}{ 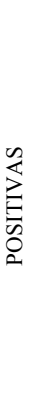 } & Alegría & 14,076 & $\begin{array}{l}, 001 * \\
*\end{array}$ & 7,873 &, $020 *$ \\
\hline & Confianza & 11,450 & $\begin{array}{l}, 003 * \\
*\end{array}$ & 5,535 &, 051 \\
\hline & Diversión & 12,425 & $\begin{array}{l}, 002 * \\
*\end{array}$ & 6,672 & ,036* \\
\hline & Orgullo & 2,736 &, 255 & 1,492 &, 474 \\
\hline & Placer & 16,476 & $\begin{array}{l}, 000 * \\
*\end{array}$ & ,402 &, 818 \\
\hline & Satisfacción & 17,344 & $\begin{array}{l}, 000 * \\
*\end{array}$ & 9,410 &, $009 * *$ \\
\hline & Simpatía & 18,693 & $\begin{array}{l}, 000 * \\
*\end{array}$ & 3,879 &, 144 \\
\hline & Tranquilidad & 11,383 & $\begin{array}{l}, 003 * \\
*\end{array}$ & 3,8436 &, 146 \\
\hline \multirow{8}{*}{ 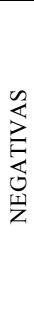 } & Aburrimiento & 18,175 & $\begin{array}{l}000^{*} \\
*\end{array}$ & 6,328 &, $042 *$ \\
\hline & Ansiedad & 4,105 &, 128 & 1,211 &, 546 \\
\hline & Desesperación & ,414 & ,813 & 2,970 & ,226 \\
\hline & Irritabilidad & 2,177 & ,337 & 1,875 & ,392 \\
\hline & Nerviosismo & 7,001 &, $030^{*}$ & 3,256 & ,196 \\
\hline & Odio & 4,105 &, 128 & 6,697 &, $035^{*}$ \\
\hline & Pesimismo & 4,894 &, 087 & 4,118 & , 128 \\
\hline & Preocupación &, 735 & 692 & 849 & ,654 \\
\hline
\end{tabular}

\section{Emociones en el aprendizaje de la Física y la Química.}

El gráfico de la Figura 3 nos muestra que los futuros profesores de la especialidad de Física/Química experimentaron en mayor porcentaje emociones positivas en el aprendizaje de la Física en Educación Secundaria. Asimismo, vemos como los sujetos de Biología/Geología experimentaron mayormente emociones negativas.

Figura 3.

Porcentaje de futuros profesores que experimentaron emociones en el aprendizaje de Física según la especialidad del Máster cursada.

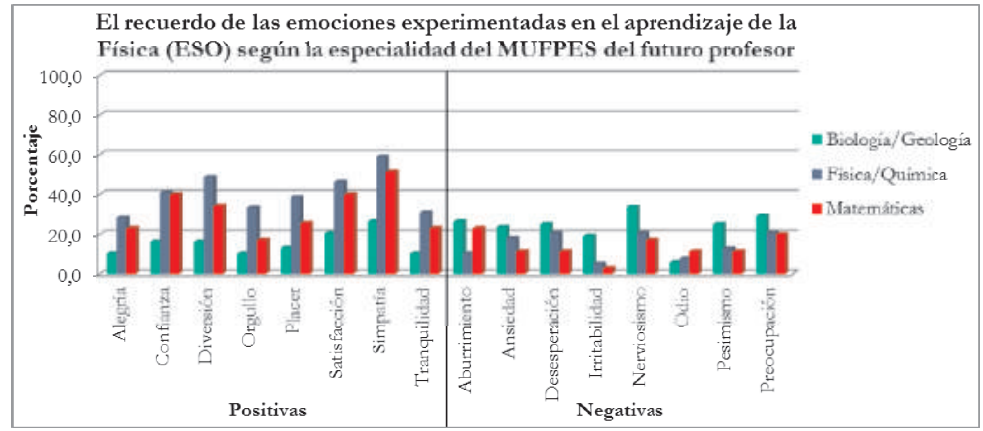




\section{LA INFLUENCIA DE LAS EMOCIONES EN LA ELECCIÓN DE CARRERAS UNIVERSITARIAS}

En el aprendizaje de contenidos de Química en Educación Secundaria, los futuros profesores de la especialidad de Física y Química experimentaron en mayor porcentaje emociones positivas, así como las emociones negativas ansiedad, irritabilidad y preocupación. En el resto de emociones negativas, la especialidad mencionada, obtiene los menores porcentajes (Figura 4).

Figura 4.

Porcentaje de futuros profesores que experimentaron emociones en el aprendizaje de Química según la especialidad del Máster cursada.

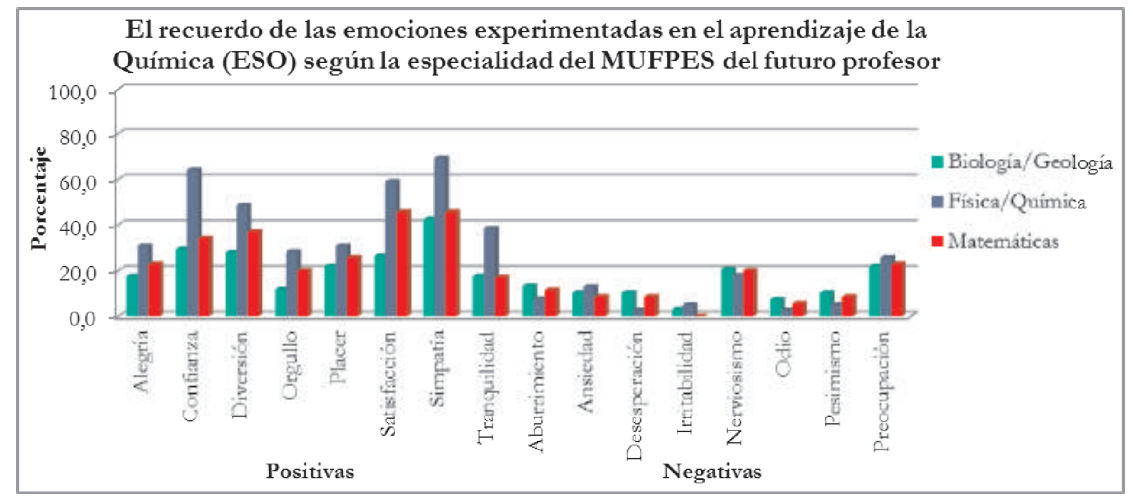

En la Tabla 5 hallamos los datos de la Prueba Chi-Cuadrado realizada entre las emociones experimentadas en el aprendizaje de la Física y la Química y las tres especialidades del Máster.

En el aprendizaje de la Física (ESO) aparecen diferencias significativas en todas las emociones positivas $(p \leq, 050)$ y en la emoción negativa irritabilidad $(p=, 017)$. Estos datos, unidos a los porcentajes que comentamos en la representación gráfica, nos indican que son los sujetos de la especialidad de Física/Química quienes experimentaron en mayor número emociones positivas al aprender Física. También, sabemos que experimentaron la emoción negativa irritabilidad en menor número.

En el aprendizaje de Química en Educación Secundaria, encontramos diferencias significativas según la especialidad del MUFPES en las emociones positivas confianza $(p=, 001)$, satisfacción ( $p$ $=, 003)$, simpatía $(p=, 024)$ y tranquilidad $(p=, 030)$, donde los profesores en formación de la especialidad de Física/Química obtienen mayores porcentajes. 
Tabla 5.

Prueba Chi-Cuadrado de la relación especialidad de Máster y el recuerdo de emociones experimentadas en el aprendizaje de Física y Química.

\begin{tabular}{|c|c|c|c|c|c|}
\hline & & \multicolumn{2}{|l|}{ Física } & \multicolumn{2}{|c|}{ Química } \\
\hline & & Chi-Cuadrado & Sig. & Chi-Cuadrado & Sig. \\
\hline \multirow{8}{*}{$\begin{array}{l}\sum_{0}^{n} \\
\vdots \\
0 \\
0\end{array}$} & Alegría & 5,958 &, $050^{*}$ & 2,448 & ,294 \\
\hline & Confianza & 10,252 & $\begin{array}{l}006^{*} \\
*\end{array}$ & 13,076 &, $001 * *$ \\
\hline & Diversión & 13,095 & $\begin{array}{l}, 001 * \\
*\end{array}$ & 4,679 & ,096 \\
\hline & Orgullo & 8,838 &, $012^{*}$ & 4,625 & ,099 \\
\hline & Placer & 9,001 &, $011^{*}$ & ,997 & ,607 \\
\hline & Satisfacción & 8,624 &, $013^{*}$ & 11,498 &, $003 * *$ \\
\hline & Simpatía & 12,649 & $\begin{array}{l}002 * \\
*\end{array}$ & 7,470 &, $024 *$ \\
\hline & Tranquilidad & 7,193 &, $027^{*}$ & 6,987 &, $030 *$ \\
\hline \multirow{8}{*}{ 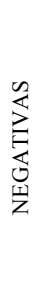 } & Aburrimiento & 3,994 & , 136 & ,763 & ,683 \\
\hline & Ansiedad & 2,237 &, 327 &, 363 &, 834 \\
\hline & Desesperación & 2,619 &, 270 & 2,117 &, 347 \\
\hline & Irritabilidad & 8,138 &, $017^{*}$ & 1,779 & ,411 \\
\hline & Nerviosismo & 4,195 &, 123 &, 111 & ,946 \\
\hline & Odio & ,995 & ,608 & 1,070 & ,586 \\
\hline & Pesimismo & 3,929 &, 140 &, 856 & ,652 \\
\hline & Preocupación & 1,597 &, 450 &, 182 & ,913 \\
\hline
\end{tabular}

${ }^{* *} \mathrm{p} \leq, 010 . \quad * \mathrm{p} \leq, 050 . \quad$ Chi-Cuadrado=Estadístico de contraste.

Sig. $=$ Significación estadística.

\section{Emociones en el aprendizaje de las Matemáticas.}

En la representación gráfica de la Figura 5 observamos claramente que son los sujetos de la especialidad de Matemáticas quienes experimentaron, en mayor porcentaje, emociones positivas en el aprendizaje de contenidos matemáticos en Secundaria. En cambio, presentan los menores porcentajes en las emociones negativas donde siempre destacan los sujetos de Biología/Geología.

Figura 5.

Porcentaje de futuros profesores que experimentaron emociones en el aprendizaje de Matemáticas según la especialidad del Máster cursada.

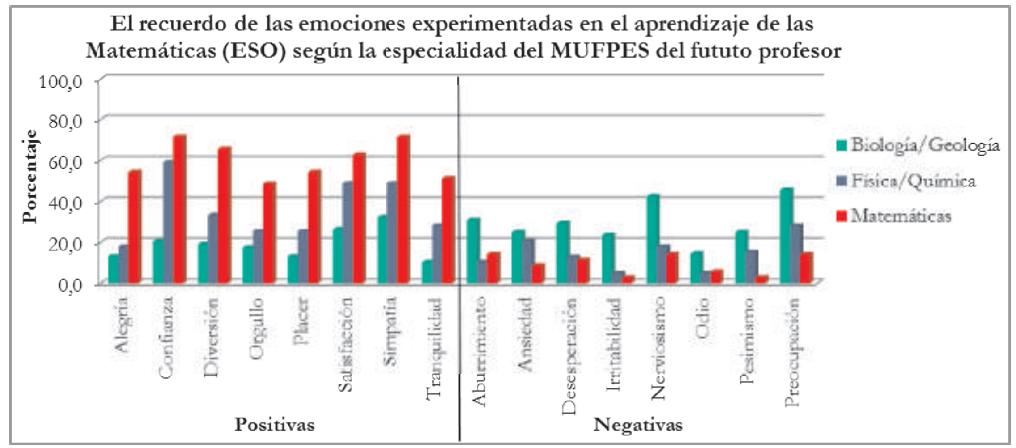




\section{LA INFLUENCIA DE LAS EMOCIONES EN LA ELECCIÓN DE CARRERAS UNIVERSITARIAS}

Los resultados de la Tabla 6, donde se recogen los datos de la Prueba Chi-Cuadrado realizada entre las emociones experimentadas en el aprendizaje de las Matemáticas (ESO) y la especialidad del MUFPES, nos indican que existe significación en todas las emociones positivas y en casi todas las emociones negativas a excepción de ansiedad $(p=, 138)$ y odio $(p=, 178)$. Por tanto, sabemos que los sujetos de la especialidad de Matemáticas experimentaron mayormente emociones positivas y en menor medida emociones negativas.

Tabla 6.

Prueba Chi-Cuadrado de la relación especialidad de Máster y el recuerdo de emociones experimentadas en el aprendizaje de Matemáticas.

\begin{tabular}{|c|c|c|c|c|c|c|c|}
\hline \multicolumn{8}{|c|}{ Matemáticas } \\
\hline & & Chi-Cuadrado & Sig. & & & Chi-Cuadrado & Sig. \\
\hline \multirow{8}{*}{ 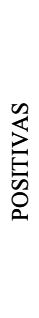 } & Alegría & 22,264 &, $000 * *$ & \multirow{8}{*}{ 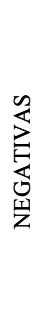 } & Aburrimiento & 7,632 &, $022 *$ \\
\hline & Confianza & 29,405 &, $000 * *$ & & Ansiedad & 3,961 & ,138 \\
\hline & Diversión & 22,232 &, $000 * *$ & & Desesperación & 6,510 &, $039 *$ \\
\hline & Orgullo & 11,182 &, $004 * *$ & & Irritabilidad & 11,679 &, $003 * *$ \\
\hline & Placer & 19,901 &, $000 * *$ & & Nerviosismo & 12,175 &, $002 * *$ \\
\hline & Satisfacción & 13,735 &, $001 * *$ & & Odio & 3,456 & ,178 \\
\hline & Simpatía & 14,291 &, $001 * *$ & & Pesimismo & 8,155 &, $017^{*}$ \\
\hline & Tranquilidad & 20,891 &, $000 * *$ & & Preocupación & 10,806 &, $005 * *$ \\
\hline
\end{tabular}

\section{CONCLUSIONES.}

Analizando las emociones experimentadas en el aprendizaje de las ciencias encontramos diferencias según la especialidad del Máster que cursan los futuros docentes.

En el aprendizaje de contenidos de Biología y Geología de Educación Secundaria, los sujetos de la misma especialidad manifiestan haber experimentado emociones positivas en mayor proporción al resto de especialidades, las cuales destacan en emociones negativas como aburrimiento, nerviosismo u odio.

Cuando aprendían las materias de Física y Química (ESO), son los sujetos de la misma especialidad quienes presentan mayoritariamente emociones positivas como confianza, satisfacción, simpatía o tranquilidad.

Y al aprender Matemáticas, un mayor número de futuro profesores de la especialidad de Matemáticas manifiesta haber experimentados emociones positivas, mientras que los sujetos de Biología/Geología destacan en casi todas las emociones negativas.

Cabe una concordancia entre las emociones experimentadas como aprendices de ciencias y las futuras carreras realizadas. Si la asignatura científica coincide con la especialidad del Máster aparecen emociones positivas, en cambio, si la materia no coincide con la especialidad surgen emociones negativas. Tal y como afirma Costillo et al. (2010), los futuros profesores de Secundaria manifiestan emociones acordes a los estudios universitarios realizados, experimentando emociones positivas en aquellas materias en las que se han especializado y negativas frente al resto.

Este resultado es muy positivo, pues si los propios profesores que tienen que impartir una materia hubiesen sentido emociones negativas hacia ella, es muy probable que esas emociones fueran transferidas a sus futuros alumnos, formando un círculo vicioso que puede repercutir en el escaso número de estudiantes matriculados en licenciaturas y grados científicos (Costillo, Borrachero, Brígido y Mellado, 2013; García y Orozco, 2008). 


\section{REFERENCIAS BIBLIOGRÁFICAS.}

Abrahams, I. (2009). Does Practical Work Really Motivate? A study of the affective value of practical work in secondary school science. International Journal of Science Education, 31(17), 23352353.

Barmby, P. y Defty, N. (2006). Secondary school pupils' perceptions of physics. Research in Science and Technological Education, 24(2), 199-215.

Bisquerra, R. (2009). Psicopedagogía de las emociones. Madrid: Síntesis.

Borrachero, A.B. y Brígido, M. (2011). Las emociones de los futuros profesores de Secundaria sobre el aprendizaje de las ciencias según el campo de procedencia. International Journal of Developmental and Educational Psychology. INFAD Revista de Psicología, 1(2), 99-106.

Brígido, M., Bermejo, M.L., Conde, M.C., Borrachero, A.B. y Mellado, V. (2010). Estudio longitudinal de las emociones en ciencias de estudiantes de maestro. Revista Galego-Portuguesa de Psicoloxía e Educación, 18(2), 161-179.

Casacuberta, D. (2000). Qué es una emoción. Barcelona: Crítica.

Costillo, E., Brígido, M., Caballero, A., Bermejo, M.L., Conde, M.C. y Mellado, V. (2010). Las emociones de futuros docentes de secundaria sobre cuestiones relacionadas con la enseñanzaaprendizaje de las ciencias. XXIV Encuentro de Didáctica de las ciencias experimentales. Organizado por la Universidad de Jaén y APICE. Baeza, 21 al 23 de julio de 2010.

Costillo, E., Borrachero, A.B., Brígido, M. y Mellado, V. (2013). Las emociones en la enseñanzaaprendizaje de las ciencias y las matemáticas de futuros profesores de Secundaria. Revista EUREKA sobre enseñanza y divulgación de las ciencias, 10(núm. extraordinario), 514-532.

Damasio, A. (2010). Y el cerebro creó al hombre. Barcelona: Editorial Destino.

Del Rincón, D., Latorre, A. y Sanz, A. (1995). Técnicas de investigación en ciencias sociales. Madrid: Dykinson.

Fensham, P. (1992). Science and technology. En P.W. Jackson (Ed.), Handbook of research on curriculum (pp.789-829). Nueva York: Macmillan Publishing Co.

Fernández-Abascal, E., Martín, M. y Domínguez, J. (2001). Procesos psicológicos. Madrid: Ediciones Pirámide.

Fouad, N.A. (2007). Work and Vocational Psychology: Theory, Research, and Applications. Annual Review of Psychology, 58, 543-64

Frijda, N. H. (2000). The psychologists' point of view. En M. Lewis y J. M. Haviland-Jones (Eds.), Handbook of emotions (pp. 59-74). New York: The Guilford Press.

García, M. y Orozco, L. (2008). Orientando un cambio de actitud hacia las Ciencias Naturales y su enseñanza en profesores de Educación Primaria. Revista Electrónica de Enseñanza de las Ciencias, 7(3), 539- 568.

Goleman, D. (1996). Inteligencia emocional. Barcelona: Cairos.

Hargreaves, A. (2000). Mixed emotions: Teachers perceptions of their interactions with students. Teaching and Teacher Education, 16(8), 811-826.

Marbá, A. y Márquez, C. (2010). ¿Qué opinan los estudiantes de las clases de ciencias? Un estudio transversal de sexto de primaria a cuarto de ESO. Enseñanza de las Ciencias, 28(1), 19-30.

Mellado, V., Blanco, J. L., Borrachero, A. B. y Cárdenas, J. A. (Eds.). (2013). Las emociones en la enseñanza y el aprendizaje de las ciencias y las matemáticas. Badajoz, España: DEPROFE.

Murphy, C. y Beggs, J. (2003). Children perceptions of school science. School Science Review, 84(308), 109-116.

Rebollo, M.A., García, R., Barragán, R., Buzón, 0. y Vega, L. (2008). Las emociones en el aprendizaje online. RELIEVE, 14(1). Recuperado en Septiembre de 2016 


\section{LA INFLUENCIA DE LAS EMOCIONES EN LA ELECCIÓN DE CARRERAS UNIVERSITARIAS}

http://www.uv.es/RELIEVE/v14n1/ RELIEVEv14n1_2.htm

Rocard, M., Csermely, P., Jorde, D., Lenzen, D., Walwerg-Henriksson, H. y Hemmo, V. (2007). Science Education Now: A Renewed Pedagogy for the Future of Europe. European Commission, Community Research.

Shapiro, S. (2010). Revisiting the teachers' lounge: Reflections on emotional experience and teacher identity. Teaching and Teacher Education, 26(3), 616-621.

Solbes, J. (2011). ¿Por qué disminuye el alumnado de Ciencias? Alambique, 17(67), 53-61.

Sutton, R. y Wheatley, K. (2003). Teachers emotions and teaching. A review of the literature and directions for future research. Educational Psychology Review, 15, 327-358.

Vázquez, A. y Manassero, M.A. (2008). El declive de las actitudes hacia la ciencia de los estudiantes: Un indicador inquietante para la educación científica. Revista Eureka sobre Enseñanza y Divulgación de las Ciencias, 5(3), 274-292.

Vázquez, A. y Manassero, M.A. (2009). La vocación científica y tecnológica: Predictores actitudinales significativo. Revista EUREKA sobre Enseñanza y Divulgación de las Ciencias, 6(2), 213-231. 